\title{
Design and Implementation of Client Side at Application Layer of Internet of Things
}

\author{
Zong-Jing Zhao, Jing-Jing Yang \\ School of Information Science and Engineering, \\ Hebei North University, \\ Zhangjiakou, Hebei, 075000, China \\ E-mail: zzj9411@foxmail.com,r78z@foxmail.com
}

\author{
Xiao Zhang, Su-Jing Ma \\ School of Information Science and Engineering, \\ Hebei North University, \\ Zhangjiakou, Hebei, 075000, China \\ E-mail: xz1965@aliyun.com,948917715@qq.com
}

\begin{abstract}
This paper designs the client side of application layer of IoT-based smart home system. Firstly, it brings forward IoT overall structure; then it compares the three short distance transmission modes of ZigBee, WIFI and Bluetooth and chooses WIFI transmission; finally it analyzes the process of connecting to server side and process of server side communication and verifies the feasibility of the plan by experiment.
\end{abstract}

\section{Keywords-iot; smart home; application layer}

\section{INTRODUCTION}

The invention of steam engines has turned over a new page of human history towards industrialization, while the appearance of the network will undoubtedly leave an impressing mark of informationization on the resplendent history of human civilization. The increasing improvement of electronic information technology and control technology, and the gradual acceleration of social informationization have promoted people's life, work, study and communication to be closely interwoven. While the informationized society is changing people's life habits and work styles, it also poses a challenge to our traditional houses. Looking around, it can be easily found that people's requirements for housing have no longer been a simple material space, but they focus more on a highly secure, comfortable, pleasant and convenient living environment, advanced communication facilities, complete and efficient information terminals, automatic and intelligent home appliances, networked resource management and shopping modes, etc. In the present society, people's increasing demands have already turned smart home into a trend [1].

Nowadays as technology is developing by leaps and bounds, Internet of Things (IoT) is regarded as a great development and reform in information field[2]. Based on a standard and interworked communication protocol, IoT connects the actual and virtual objects through network; through information exchange and communication, IoT realizes intelligent identification, positioning, tracing, monitoring and management. Smart home system is a control system that integrates home communication, equipment auto control, security protection, etc, through Internet; in other words, it connects various home appliances, security devices and measuring equipment to form an internal home network, centrally managed by intelligent controller (central control unit). So to speak, smart home system is an outcome of the combination of IoT and people's modern living demands [3].
The key factors of IoT development include a three-layer architecture, namely, perception layer, network layer and application layer (as shown in Figure 1)[4]. Perception layer realizes intelligent perceiving and identification of the physical world, information collecting and processing and auto control, and connects physical entities to network layer and application layer through communication modules. Network layer mainly realizes information transfer, routing and control, including extended network, access network and core network; network layer can depend on public telecommunication network and Internet, as well as specialized industrial communication network. Application layer includes application infrastructure/middleware and various IoT apps; application infrastructure/middleware provides general basic service facilities for information processing and computing, calling interfaces of ability and resources for IoT apps, and based on this, IoT is applied in multiple fields. This paper focuses mostly on the design and implementation of client side at application layer of smart home system.

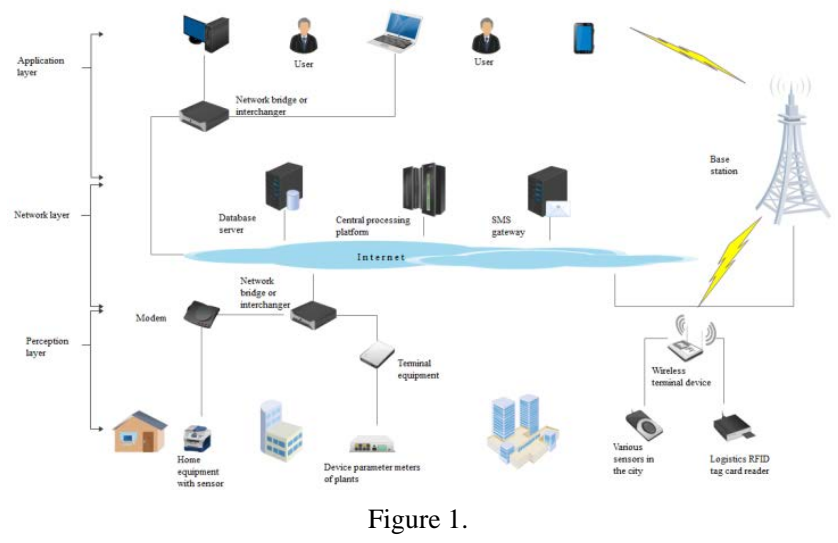

\section{DESIGN OF SYSTEM'S APPLICATION LAYER}

\section{A. Function Analysis}

At present stage, smart home constructs an efficient management system for housing facility and home daily routine, integrating the facilities related to home life by means of comprehensive wiring technology, network communication technology, security prevention technology, automatic control technology and audio/video technology with a house as the platform; it can improve the safety, convenience, comfort and artistry of home life and realize 
an environment-friendly and energy-saving living condition. In the application layer of IoT, the functions fall into the following six basic categories:

- Monitoring alarm

- Date collecting

- Information push
- Video monitoring

- Remote control

- Identification and positioning

With the two points combined and the functions categorized, we make the following table:

TABLE 1

\begin{tabular}{|c|c|c|c|c|c|c|}
\hline Ability indicator & Monitoring alarm & Date collecting & Information push & Video monitoring & Remote control & $\begin{array}{c}\text { Identification and } \\
\text { positioning }\end{array}$ \\
\hline Upload flow & No & Smaller data size & Small data size & Large data size & Smaller data size & Smaller data size \\
\hline Download flow & Smaller data size & Larger data size & Larger data size & Large data size & Small data size & Smaller data size \\
\hline $\begin{array}{l}\text { Application } \\
\text { direction }\end{array}$ & $\begin{array}{l}\text { Home security } \\
\text { system }\end{array}$ & $\begin{array}{l}\text { Home environment } \\
\text { monitoring }\end{array}$ & $\begin{array}{l}\text { Home using status } \\
\text { confirmation }\end{array}$ & $\begin{array}{l}\text { Building intercom } \\
\text { system }\end{array}$ & $\begin{array}{l}\text { Mobile terminal } \\
\text { controlled home } \\
\text { appliances }\end{array}$ & $\begin{array}{l}\text { Mobile terminal } \\
\text { connected home } \\
\text { appliances }\end{array}$ \\
\hline
\end{tabular}

\section{B. Analysis of Wireless Transmission}

Internationally, wireless network transmission protocol is a wireless transmission protocol the same as infrared, Bluetooth, GPRS[5], CDMA1X protocols. At present, the wireless transmission protocols used in IoT include WIFI, GPRS, infrared technology, ZigBee technology, Bluetooth technology and RFID technology. In the last several meters of IoT that connects Internet and things, short distance wireless transmission technology plays the pivotal role. At present, there are various short distance wireless transmission technologies which can be used in IoT. In China, besides RFID technology which has been widely applied, there are also relatively mature technologies such as WIFI, ZigBee, Bluetooth, etc., and the new technologies deriving from the above-mentioned technologies. These technologies have their own features; due to their different requirements for transmission speeds, distances, power consumptions, etc., they have formed respective IoT application scenarios. As smart home system adopts short distance transmission, we make a comparison of the three short distance transmission technologies, including ZigBee, WIFI and Bluetooth:

1) ZigBee: ZigBee has received extensive attention in IoT due to its distinct technical features. The frequency bands used by ZigBee are respectively $2.4 \mathrm{GHz}, 868 \mathrm{MHz}$ (Europe) and $915 \mathrm{MHz}$ (U.S.). Its main technical features are: firstly, low data transmission rate, only at 10Kbps-250Kbps; secondly, low power consumption caused by low transmission rate, with low transmitting power only at $1 \mathrm{~mW}$, and it is estimated that ZigBee can last a service time of 6 months to 2 years with only two double A batteries - this is a unique advantage of ZigBee; thirdly, low cost due to its low transmission rate and simple protocol; fourthly, large network capacity: each ZigBee network can support 255 devices to the maximum; in one area there can be at most 100 ZigBee networks simultaneously and the newwork formation is flexible.

2) WiFi: WIFI is a wireless ethernet extension technology.
If multiple users have access to a hotspot, the bandwidth will be shared by these users and WIFI rate will drop. WIFI signals in $2.4 \mathrm{GHz}$ frequency band are slightly affected by wall obstructions. [6] WIFI transmission rate is continuously improving as technology evolves. In China, the WIFI technology adopted by telecom operators for constructing wireless cities has upgraded to 802.11n and the highest rate has risen from $11 \mathrm{Mbps}$ of $802.11 \mathrm{~g}$ standard to above 50Mbps.

3) Bluetooth: Bluetooth can exchange wireless information between numerous devices such as mobiles, PDA, wireless earphones, notebooks and related peripheral devices. Bluetooth adopts distributed network structure, fast frequency hopping and short packet technology, supports point-to-point and point-to-multipoint communication, and operates in universal $2.4 \mathrm{GHz}$ frequency band in the globe; its data rate is $1 \mathrm{Mbps}$.

WIFI has high transmission rate and long effective distance. Wireless fidelity technology is a short distance wireless technology suitable for office and home uses; it suits best the requirements of smart home system for wireless transmission modes. This system adopts WIFI for the data transmission between server side and client side.

\section{IMPLEMENTATION PLAN}

\section{A. Connection Process}

In the client side of the application layer of IoT-based smart home system, connection to the specific server side is a key step. The concrete process is as follows in Figure 2:

First input the IP address and port number of target server side for connection; if having successfully logged in, the operator can access operation interface for operations, otherwise he/she has to return to login interface to try again.

\section{B. Communication Process}

At operation interface, it's required to issue user's command to server side and meanwhile to process the data returned by server side and display it in front of the user. 
The concrete process is as follows in Figure 3:

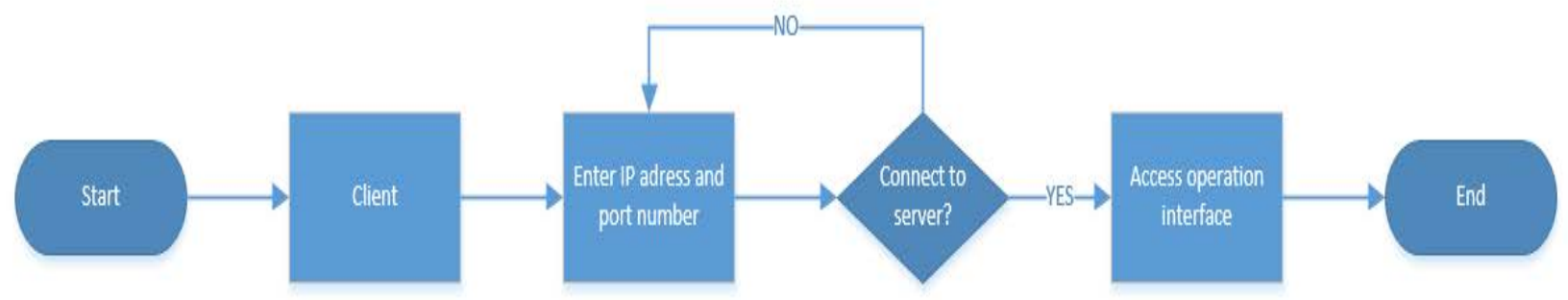

Figure 2

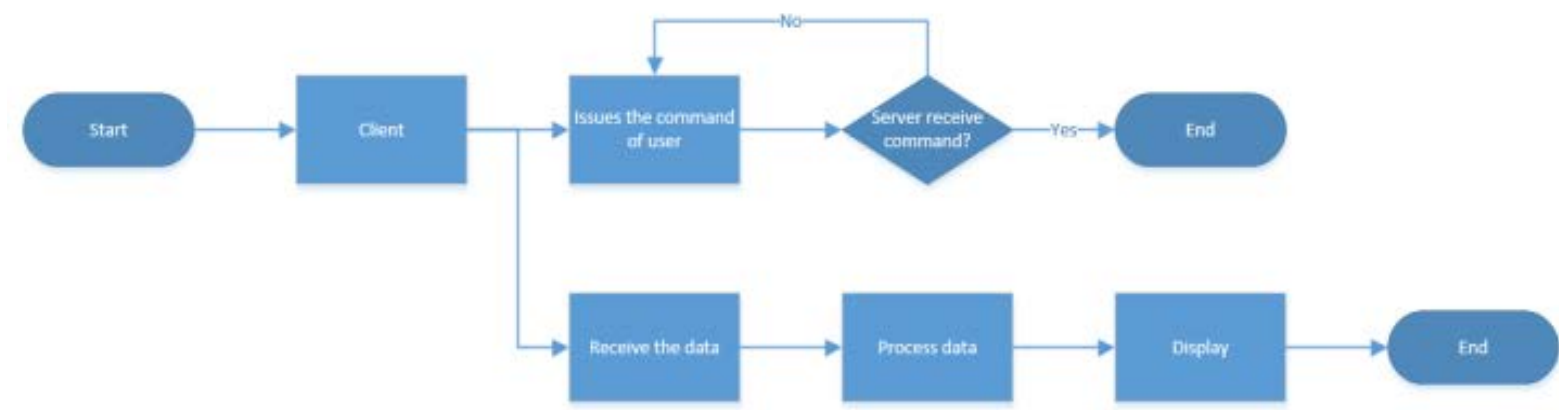

Figure 3

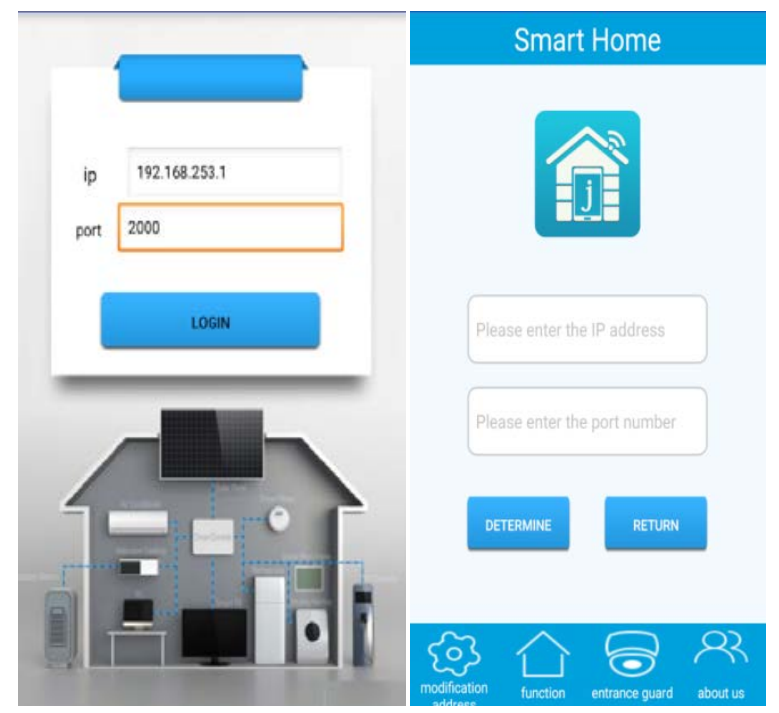

Figure 4

\section{EXPERIMENT}

According to the design and plan of the client side of application layer of smart home system, server side of high performance is adopted, Android Studio 2.1.2 is used as the development tool for client side app, and a mobile of Android 6 system is used as the test platform of client side app. The experiment result is as shown in the above Figure 4.

The two pictures in Figure 4 are the login interface that requires IP address and port number for initializing and the interface for modifying IP address and port number.

The above figure is an example of the environment monitoring parameters sent from server side, like temperature, humidity and PM; air conditioning and lighting are an example of the status of the electric appliances at present and meanwhile the electric appliances can be controlled; the status of smoke alarm is also obtained to judge if it's proper to give an alarm.

In the above figure, the building monitoring parameters can realize the video talkback and control between mobile terminal and building. 


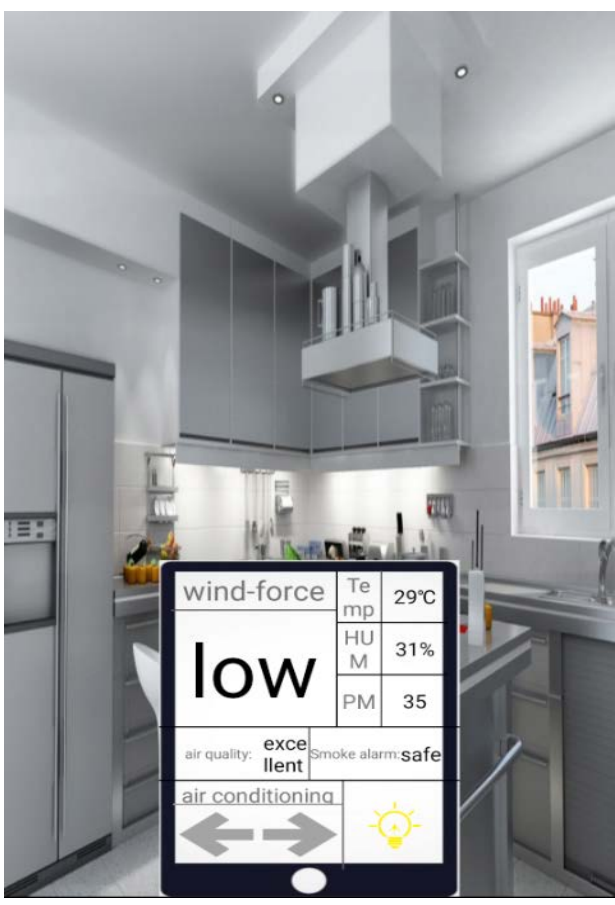

Figure 5

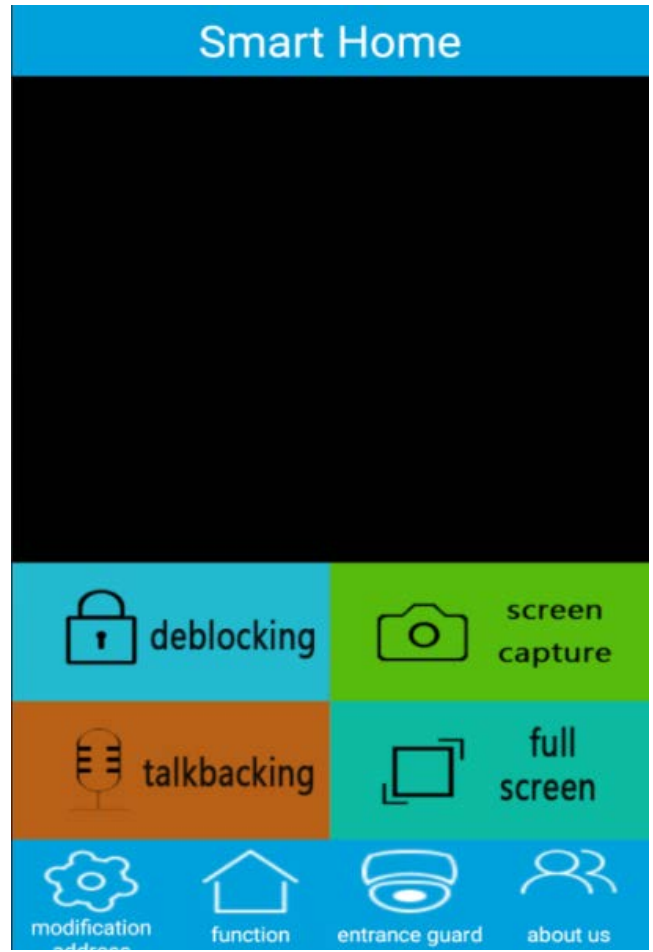

Figure 6

\section{CONCLUSION}

This paper designs the client side of application layer of IoT-based smart home system. Firstly, it brings forward IoT overall structure; secondly, it analyzes its function; then it compares the three short distance transmission modes of ZigBee, WIFI and Bluetooth and chooses WIFI transmission; finally it analyzes the process of connecting to server side and process of server side communication and verifies the feasibility of the plan by experiment.

\section{ACKNOWLEDGEMENTS}

The work of this paper was supported by the Population Health Informatization in Hebei Province Engineering Technology Research Center and Medical Informatics in Hebei Universities Application Technology Research and Development Center.

\section{REFERENCES}

[1] Friedewald, Michael, et al. "Perspectives of ambient intelligence in the home environment." Telematics and informatics 22.3 (2005): 221-238.

[2] TongKe, Fan. "Smart Agriculture Based on Cloud Computing and IOT." Journal of Convergence Information Technology 8.2 (2013).

[3] Chan, Marie, et al. "Smart homes-current features and future perspectives." Maturitas 64.2 (2009): 90-97.

[4] Atzori, Luigi, et al. "The social internet of things (siot)-when social networks meet the internet of things: Concept, architecture and network characterization." Computer Networks 56.16 (2012): 3594-3608.

[5] P Pöyhönen. "System and method for establishing a session initiation protocol communication session with a mobile terminal." US Patent (2015): 8,989,737.

[6] S Shao, A Khreishah, $M$ Ayyash. "Design and analysis of a visible-light-communication enhanced WIFI system ." Journal of Optical (2015) 